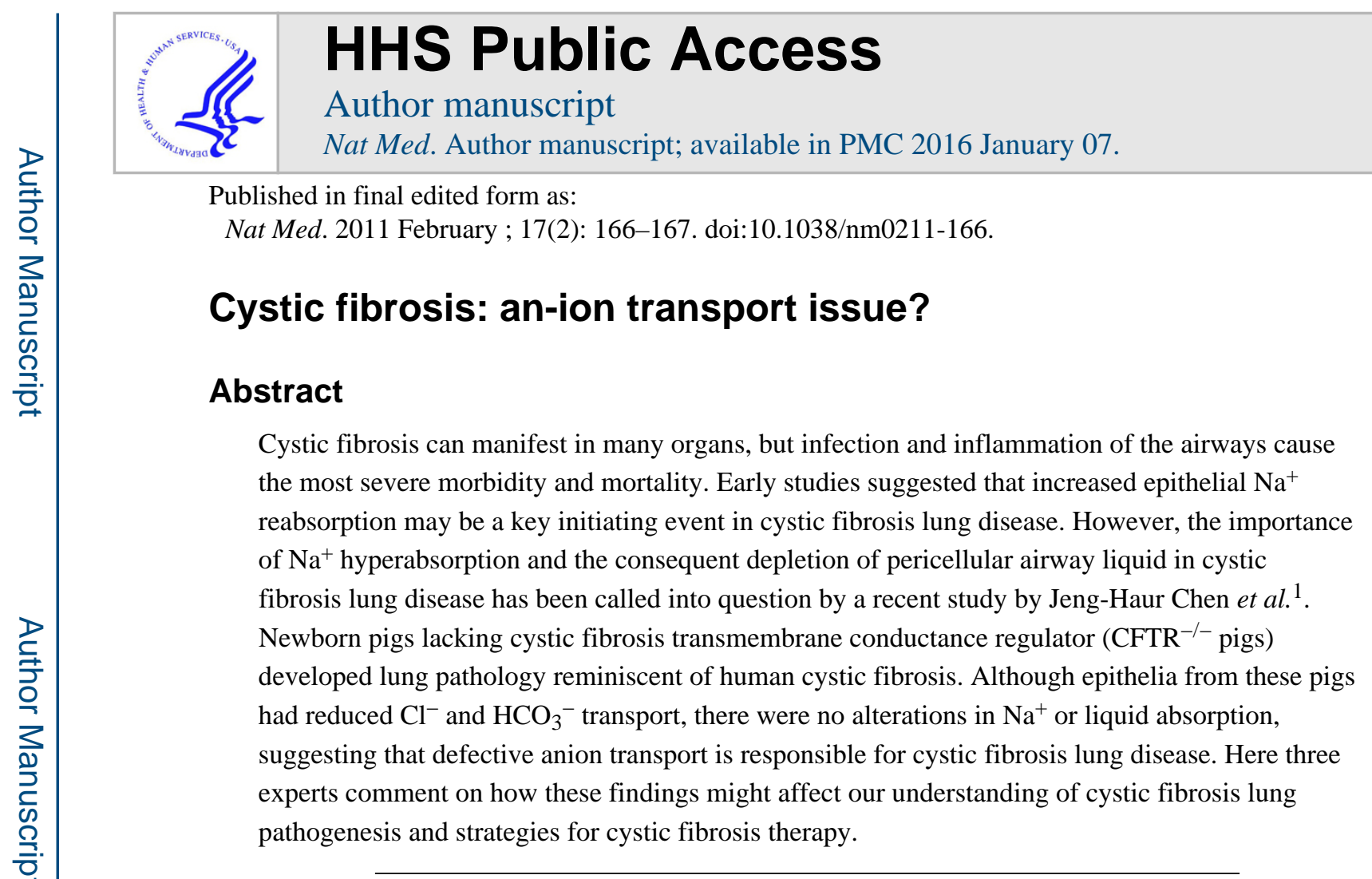

\title{
Gerald Pier
}

The major clinical manifestation of cystic fibrosis-chronic and life-shortening lung infections - could be considered an iatrogenic disease caused by medical intervention. Cystic fibrosis was first identified clinically as a fatal disease of young children resulting from gastrointestinal and secretory organ dysfunction. Defective transepithelial ion transport has long been recognized to be a major contributor to cystic fibrosis symptoms, and the identification of the CFTR gene and protein allowed mechanistic links to be made between nonfunctional or missing CFTR and defects in transport of $\mathrm{Cl}^{-}$and $\mathrm{HCO}_{3}{ }^{-}$. People with cystic fibrosis now survive to 30-40 years of age, as they can be treated with therapies to correct gastrointestinal dysfunction, but they suffer from chronic lung infections for most of their life. This lung disease is also thought to be due to defective transepithelial ion transport of $\mathrm{Cl}^{-}$and $\mathrm{HCO}_{3}{ }^{-}$, as well as of $\mathrm{Na}^{+}$, whose absorption from the airway lumen has been suggested to be affected by CFTR.

Chen et al. ${ }^{1}$ have now added a wrinkle to the area of cystic fibrosis research by finding that $C F T R^{-/}$pig lungs were not affected in their ability to transport $\mathrm{Na}^{+}$and absorb water. They also observed no reduction in the volume of pericellular airway liquid ${ }^{1}$, which is thought to be key for clearing bacteria. However, these observations do not explain how defective ion transport in the lung is connected to the major clinical manifestation of modern cystic fibrosis - chronic lung infection with the mucoid variant of Pseudomonas aeruginosa ${ }^{2}$. Clearly, CFTR-dependent defects in innate immunity are major factors in the pathogenesis of cystic fibrosis lung disease ${ }^{3}$. Whereas cystic fibrosis pigs showed defective antibacterial clearance $^{4}$, it is not known whether they would develop P. aeruginosa infections as humans with cystic fibrosis do. An important challenge is to connect electrophysiologic defects in cystic fibrosis with chronic $P$. aeruginosa infection. 
In addition, as major clinical therapies are directed toward corrector and potentiator drugs for enhancing the amount and function of mutant CFTR proteins in lung cells, this study suggests that the effects of these drugs on ion transport may need to be reevaluated if $\mathrm{Na}^{+}$ transport is not defective in human cystic fibrosis.

\section{Professor of Medicine (Microbiology and Molecular Genetics), Harvard Medical School and Brigham and Women's Hospital, Boston, Massachusetts, USA.}

\section{COMPETING FINANCIAL INTERESTS}

The author declares no competing financial interests.

\section{Alice Prince}

Since its original description in infants with excessively salty sweat, cystic fibrosis has been recognized as a disease of defective electrolyte transport. Much less clear is how a defective chloride channel results in pulmonary infection and eventual respiratory failure. The recently developed $\mathrm{CFTR}^{-/-}$pig may provide a model to determine how CFTR dysfunction affects mucosal immunity. Like their human counterparts, the $C F T R^{-/-}$pigs have significantly increased spontaneous bacterial colonization of their airways and a diminished ability to clear Staphylococcus aureus ${ }^{4}$. Diverse organisms are found in the neonatal $\mathrm{CFTR}^{-/-}$pig airway, as expected early in the colonization process before predominant species emerge.

Chen et al. ${ }^{1}$ showed that this increased bacterial colonization occurs in the $C F T R^{-/-}$pigs at a time when there is no evidence of excessive $\mathrm{Na}^{+}$reabsorption or depletion of the periciliary fluid volume in the airways. The $\mathrm{CFTR}^{-/-}$pigs get infected despite adequately hydrated airway secretions, challenging the long-accepted dogma that infection in cystic fibrosis is a consequence of dehydrated mucus, which is responsible for airway obstruction and subsequent selection of genetically versatile bacteria that adapt to this milieu.

These findings do not necessarily negate the clinical utility of improving airway hydration, especially in the presence of infection. Hydrating airway secretions, along with antibiotic therapy, have been mainstays of cystic fibrosis therapy dating back to the mist tents used in the 1960s, and ongoing clinical trials are testing drugs to block $\mathrm{Na}^{+}$and water reabsorption. Even if these hydration strategies do not target the primary cause for increased susceptibility to infection in cystic fibrosis, they still may enhance mucociliary clearance.

Together, the data presented by Chen et al. ${ }^{1}$ and Stoltz et al. ${ }^{4}$ provide new impetus to establish whether there is an inherent mucosal immune defect responsible for bacterial colonization of the cystic fibrosis lung, beyond obstruction due to mucus plugging. CFTRassociated abnormalities in epithelial type I interferon and nuclear factor- $\kappa \mathrm{B}$-dependent signaling cascades that affect the recruitment and activation of immune cells have been well described in vitro. The $\mathrm{CFTR}^{-/-}$pig provides an appropriate model to determine whether these or other immune abnormalities result in the impaired bacterial clearance observed by Stoltz et al. ${ }^{4}$. The $\mathrm{CFTR}^{-/-}$pig will also be useful in establishing whether early pharmacological correction of CFTR dysfunction—using drugs that are currently in clinical 
trials - can prevent infection, whether or not dehydrated airway secretions are a primary or secondary effect of this disease.

\section{Professor of Pediatrics (in Pharmacology), College of Physicians \& Surgeons, Columbia University, New York City, New York, USA.}

\section{COMPETING FINANCIAL INTERESTS}

The author declares no competing financial interests.

\section{André Cantin}

Almost 30 years after the observation that the nasal epithelial surfaces of people with cystic fibrosis have increased amiloride-sensitive negative voltage (Vt-amiloride) ${ }^{5}$, a key question remains-is lung disease initiated by excessive sodium uptake and liquid hyperabsorption or by defective transepithelial anion transport? The answer has important therapeutic implications for infants with cystic fibrosis in whom early intervention may prevent irreversible airway damage.

Mice overexpressing the $\beta$-subunit of the epithelial $\mathrm{Na}^{+}$channel (ENaC) show decreased airway surface liquid volume, abnormal mucus transport and delayed bacterial clearance. The latter two are key features of cystic fibrosis lung disease ${ }^{6}$. Furthermore, in these mice the cystic fibrosis-related increase in transepithelial nasal potential difference can be restored by the $\mathrm{ENaC}$ inhibitor amiloride.

But, are sodium hyperabsorption and airway surface liquid depletion necessary to initiate cystic fibrosis disease? According to Chen et al. ${ }^{1}$, the answer is no, at least in the newborn $C_{F T R^{-/-}}$pig model. The recently developed $C_{F T R^{-/}}$pig reproduces the lung and digestive tract features of cystic fibrosis and provides a new window through which to view early events preceding airway inflammation. The $C F T R^{-/-}$pig has an increase in nasal Vtamiloride similar to that observed in humans with cystic fibrosis. However, $\mathrm{CFTR}^{-/-}$pig nasal tissues do not show increased $\mathrm{Na}^{+}$conductance, and their airway surface liquid volume is normal. The changes in Vt-amiloride can be entirely explained by defective anion conductance of chloride and bicarbonate. Therefore, decreased CFTR-dependent anion conductance alone is associated with a phenotype similar to that of increased $\mathrm{Na}^{+}$and airway surface liquid absorption.

What are the implications for people with cystic fibrosis? These studies were performed exclusively in newborn pigs. It is conceivable that $\mathrm{ENaC}$ function and airway surface liquid volume vary between nasal and bronchial tissues, between species, during inflammation and infection, and with age. Furthermore, because $\mathrm{Na}^{+}$hyperabsorption and airway surface liquid depletion lead to cystic fibrosis-like airway changes, hydration of the airway surface liquid may be of therapeutic benefit. However, we now know that increased $\mathrm{Na}^{+}$ conductance and decreased airway surface liquid volume are not necessary to induce cystic fibrosis-like changes in newborn airways. This new information should sharply focus our attention on issues of defective anion conductance in cystic fibrosis airway pathophysiology, such as the role of bicarbonate anions defining mucus viscosity ${ }^{7}$. Most important, new 
therapies for infants with cystic fibrosis should be directed toward the correction of defective CFTR-dependent anion conductance.

\section{Director of the Pulmonary Physiopathology Research Axis of the Clinical Research Center Etienne Le-Bel, Centre hospitalier universitaire de Sherbrooke, Sherbrooke, Quebec, Canada.}

\section{COMPETING FINANCIAL INTERESTS}

The author declares no competing financial interests.

\section{References}

1. Chen JH, et al. Loss of anion transport without increased sodium absorption characterizes newborn porcine cystic fibrosis airway epithelia. Cell. 2010; 143:911-923. [PubMed: 21145458]

2. Farrell PM, et al. Association between mucoid Pseudomonas infection and bronchiectasis in children with cystic fibrosis. Radiology. 2009; 252:534-543. [PubMed: 19703887]

3. Campodonico VL, Gadjeva M, Paradis-Bleau C, Uluer A, Pier GB. Airway epithelial control of Pseudomonas aeruginosa infection in cystic fibrosis. Trends Mol Med. 2008; 14:120-133. [PubMed: 18262467]

4. Stoltz DA, et al. Cystic fibrosis pigs develop lung disease and exhibit defective bacterial eradication at birth. Sci Transl Med. 2010; 2:29ra31.

5. Knowles M, Gatzy J, Boucher R. Relative ion permeability of normal and cystic fibrosis nasal epithelium. J Clin Invest. 1983; 71:1410-1417. [PubMed: 6853720]

6. Mall M, Grubb BR, Harkema JR, O’Neal WK, Boucher RC. Increased airway epithelial $\mathrm{Na}^{+}$ absorption produces cystic fibrosis-like lung disease in mice. Nat Med. 2004; 10:487-493. [PubMed: 15077107]

7. Chen EY, Yang N, Quinton PM, Chin WC. A new role for bicarbonate in mucus formation. Am J Physiol Lung Cell Mol Physiol. 2010; 299:L542-L549. [PubMed: 20693315] 


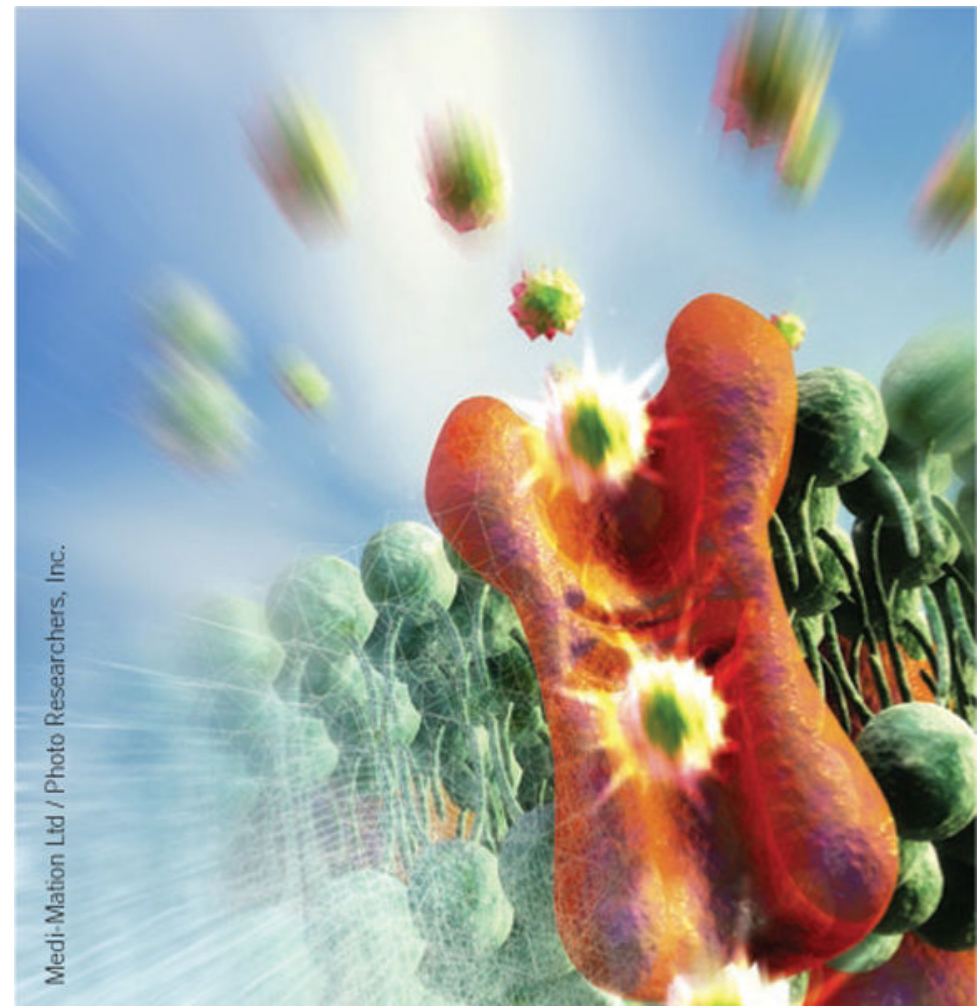

Defective anion transport may be the initiating event in cystic fibrosis lung disease. 